\title{
Young's Modulus Measurement of Metal Wires Using FBG Sensor
}

\author{
Chunchan CHEN, Li LIANG, Wenwen QUAN, Liangzhang LIAO, and Junhui HU* \\ College of Physical Science and Technology, Guangxi Normal University, Guilin 541004, China \\ *Corresponding author: Junhui HU_ E-mail: hujh@mailbox.gxnu.edu.cn
}

\begin{abstract}
A novel Young's modulus measurement scheme based on fiber Bragg gratings (FBG) is proposed and demonstrated experimentally. In our method, a universal formula relating the Bragg wavelength shift to Young's modulus is derived and metal wires are loaded strain by using the static stretching method. The Young's modulus of copper wires, aluminum wires, nickel wires, and tungsten wires are separately measured. Experimental results show that the FBG sensor exhibits high measurement accuracy, and the measurement errors relative to the nominal value is less than $1.0 \%$. The feasibility of the FBG test method is confirmed by comparing it with the traditional charge coupled device (CCD) imaging method. The proposed method could find the potential application in the material selection, especially in the field that the size of metal wires is very small and the strain gauges cannot be qualified.
\end{abstract}

Keywords: Fiber Bragg grating; Young's modulus; static stretching method; metal wire

Citation: Chunchan CHEN, Li LIANG, Wenwen QUAN, Liangzhang LIAO, and Junhui HU, "Young's Modulus Measurement of Metal Wires Using FBG Sensor," Photonic Sensors, 2019, 9(3): 277-283.

\section{Introduction}

Fiber Bragg grating (FBG) sensor has been used in a large number of sensing applications due to its high sensitivity, light weight, small size, immunity to external electromagnetic disturbance, and the ability to function in harsh environment [1]. The basic principle of FBG sensor is based on the fact that the Bragg wavelength shifts with the change of external environment parameters due to the thermo-optic effect and strain effect. FBG sensor exhibits all of the benefits associated with other optical fiber sensors, especially their ability to be multiplexed [2, 3]. Many different FBG sensors have been developed for measuring strain [4], temperature $[5,6]$, pressure [7], refractive index [8, 9], curvature [10], pressure [11], and shock stress [12].

Young's modulus is significant for physical parameter of solid material, which describes the physical capacity of the solid material resistance to deformation. Measuring accurately Young's modulus of solid materials is crucial to the material selection for civil engineering, machine design, and development of new materials. Current methods for measurement of Young's modulus can be divided into the optical method and the electrical method [13, 14]. Optical lever, charge coupled device (CCD) imaging system, and position sensitive detector (PSD) are often used in optical methods, and strain gauge and Hall position sensors are mainly applied in electrical methods at present. Recently, engineering measurement for Young's modulus change based on 3-point bending test was reported. 
In their measurements, Young's modulus is determined by the deformation of the specimen by using three laser displacement meters with a precision of $1 \mu \mathrm{m}$. The strains at $30 \%$ and $5 \%$ of the maximum load are used for calculating Young's modulus [15]. However, this method requires a relatively complex system.

In our previous work, we have demonstrated measuring Young's modulus of metal beams by using fiber Bragg gratings (FGBs) based on the three-point bend testing method [16]. In this paper, we propose and demonstrate experimentally a high-accuracy Young's modulus measurement of metal wires by using the FBG sensor based on the static stretching method. Besides the different testing methods, another important difference is that the test object is a small metal wire with the diameter in the magnitude of $0.1 \mathrm{~mm}$, in which the strain gauges cannot be qualified. In the experiment, Young's moduli of four metal wires are measured by the proposed scheme. The experimental results with FBG sensor exhibits high measurement accuracy, and the measurement errors relative to the nominal value are less than $1.0 \%$. The feasibility of the FBG test method is also confirmed by comparing it with the traditional CCD imaging method.

\section{Experimental setup and measurement principle}

The scheme of experiment is shown in Fig. 1. The light from an amplified spontaneous emission (ASE) broadband light source $(20 \mathrm{dBm}$, $1525 \mathrm{~nm}-1565 \mathrm{~nm}$ ) is launched into the FBG via an optical circulator. And then, the reflected spectrum of FBG which includes the tensile deformation information of the wire is recorded by an optical spectrum analyzer (OSA, YOKOGAWA, AQ6370C) with the resolution of $0.02 \mathrm{~nm}$. Because the diameter of the wire is only $0.1 \mathrm{~mm}$ order of magnitude, the conventional FBG attached method is unsuitable to our measurement [11]. In order to guarantee the strain of the wire is fully transferred to the FBG as much as possible, two ends of FBG are fixed on the wire by fusion splicing using two heat-melt tubes
(HMTs) with a commercial splicer (Fujikura FSM-60S). One end of the wire to be measured is fixed, and the other end hangs a tray for adding or reducing the scales to realize static stretching.

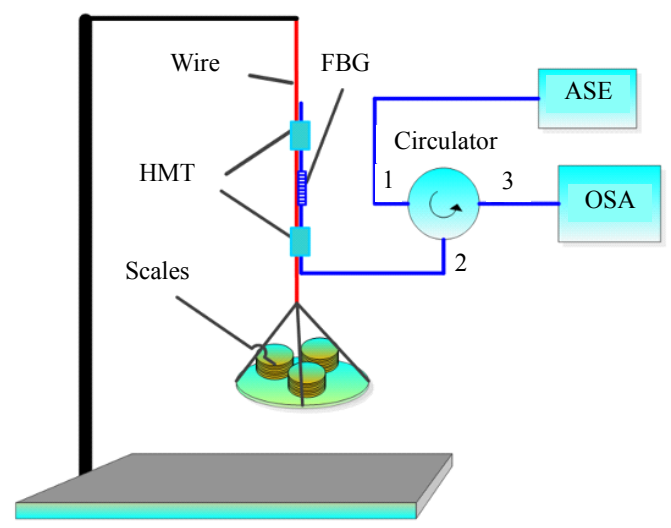

Fig. 1 Experimental setup of Young's modulus measurement based on FBG.

It is well known that the Bragg wavelength of FBG is satisfied as [16]

$$
\lambda_{B}=2 n_{\mathrm{eff}} \Lambda
$$

where $\lambda_{B}$ is the Bragg wavelength, $n_{\text {eff }}$ is the effective refractive index of the core of FBG, and $\Lambda$ is the period of FBG. According to this equation, it is known that the Bragg wavelength shifts when the period or the effective refractive index changes. All the measurements are carried out in an air-conditioned laboratory where the temperature is set at $25^{\circ} \mathrm{C}$. Consequently, the impact of temperature change on the Bragg wavelength shift is negligible. Then the Bragg wavelength shifts with the axial strain of FBG can be expressed as [16]

$$
\begin{gathered}
\Delta \lambda_{B}=\left\{1-n_{\text {eff }}^{2}\left[P_{12}-\mu\left(P_{12}+P_{11}\right)\right] / 2\right\} \varepsilon_{z B} \lambda_{B} \\
\Delta \lambda_{B}=k_{z} \varepsilon_{z B} \lambda_{B}
\end{gathered}
$$

where $P_{11}$ and $P_{12}$ are the Pockels coefficients of the photoelastic tensor, of which the values are 0.12 and 0.27 , respectively [18]. The Poisson coefficient $\mu$ of optical fiber is typically 0.17 . And the typically strain sensitivity $k_{z}$ is 0.784 [19]. $\varepsilon_{z B}$ is the strain loaded on the FBG. It is obvious that the relative shift of Bragg wavelength is linearly related to the axial strain based on (3). When the axial stress is imposed to the wire by adding scales in the tray, the 
tensile elongation of the wire will transmit to the FBG. Therefore, the axial strain of the wire can be obtained by measuring the relative Bragg wavelength shift.

According to Hooke's law, when the wire encounters elastic deformation by external force, the system satisfies

$$
F / S=E \cdot \varepsilon_{z}
$$

where $S, \varepsilon_{z}$, and $E$ are the cross-sectional area, axial strain, and Young's modulus of the wire, respectively. $d$ is the diameter of the wire, and $F$ is the axial force applied on the wire. It must be pointed out that the gravity force of scales is shared by the wire and the FBG when the diameter of testing metal wires is comparable to FBG. And the axial force applied on the FBG is half of the gravity force. In other words, the axial force $F$ in (4) is approximately equal to the half of the scales gravity force. Because the two ends of FBG are fixed on the wire by the HMTs, it is obvious that the tensile elongations of the FBG and the wire between the two HMTs are equal. It indicates that the strain loaded on FBG is equal to that on the wire, i.e. $\varepsilon_{z B}=\varepsilon_{z}$. Therefore, Young's modulus of the wire can be expressed as follows:

$$
E=\left[\left(4 k_{z} \lambda_{B}\right) /\left(\pi d^{2}\right)\right] \cdot\left(F / \Delta \lambda_{B}\right) .
$$

In the experiment, once the ratio (or its reciprocal) of applied axial force $F$ and the shift of Bragg wavelength $\Delta \lambda_{B}$ is measured, the Young's modulus of the wire can be calculated.

\section{Results and discussion}

\subsection{Young's modulus measurement of copper wires}

The FBG reflected spectra shift with the change of the applied axial force $F_{z B}$ without wire is measured firstly. The laboratory temperature is kept at $25^{\circ} \mathrm{C}$ controlled by an air conditioner during the whole experimental process. Therefore, the effect of temperature on the shift of Bragg wavelength $\Delta \lambda_{B}$ can be negligible. According to Fig. 2, it shows excellent linear properties between the axial force
$F_{z B}$ and Bragg wavelength shifts, which is $F_{z B}=1.357 \times \Delta \lambda_{B}-0.0006$, and the $R^{2}$ value is 0.999 .

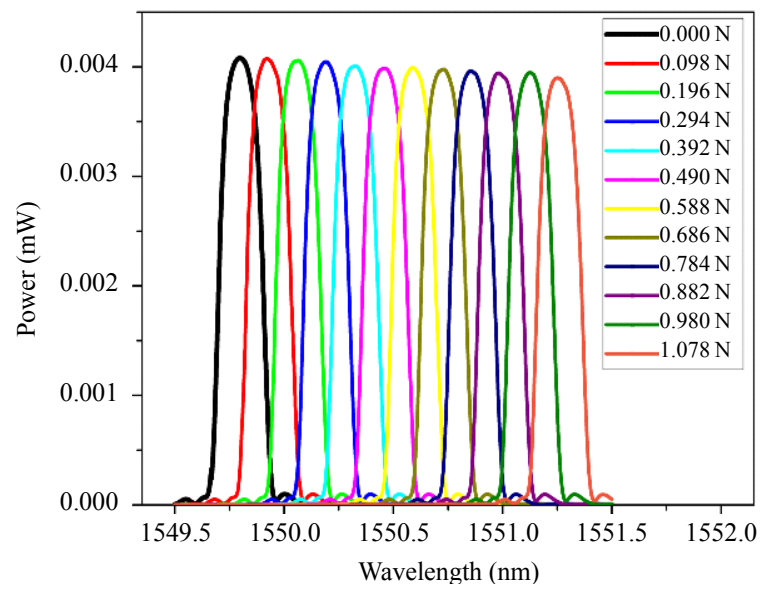

(a)

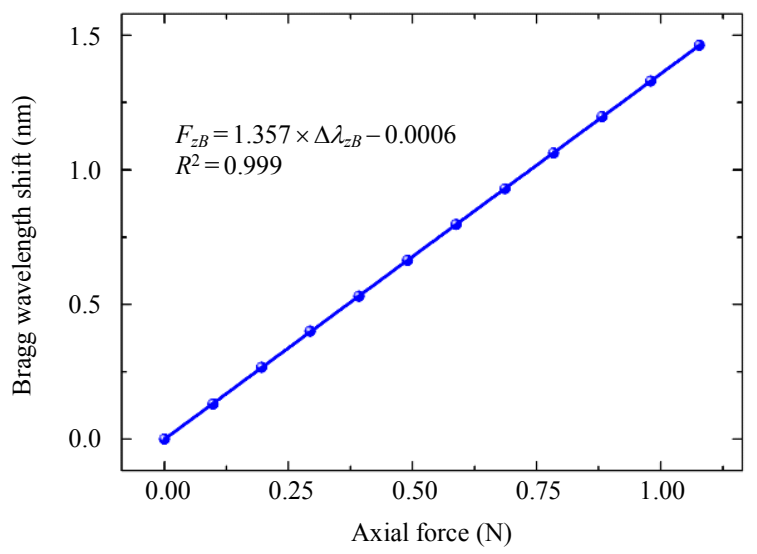

(b)

Fig. 2 Bragg wavelength of bare FBG shifts with the loaded axial force: (a) the measured FBG reflected spectra shifts with the change of the applied axial force $F_{z B}$ without wire and (b) the Bragg wavelength of FBG responses to the variation of the loaded axial force without wire.

Young's modulus measurement of copper wire is then measured, and the feasibility and repeatability of the method is also experimentally validated. In the experiments, a section of $1 \mathrm{~m}$ long copper wire with a diameter of $0.18 \mathrm{~mm}$ is used. The nominal Young's modulus in data sheet is $110.00 \mathrm{GPa}$. The Bragg wavelength of the FBG is $1549.934 \mathrm{~nm}$ at room temperature of $25^{\circ} \mathrm{C}$, reflectivity is $92.5 \%$, and spectral width $(3 \mathrm{~dB})$ is about $0.18 \mathrm{~nm}$. The ratio of applied axial force $F$ (half of the scales gravity force) and the shift of Bragg wavelength $\Delta \lambda_{B}$ are measured by applying axial force $F$ from $0 \mathrm{~N}$ to $1.86 \mathrm{~N}$ with a step about $0.30 \mathrm{~N}$. The results are 
plotted in Fig. 3. The measured FBG reflected spectra show a uniform spacing change with an increase in the applied axial force [see Fig.3(a)], which well agrees with the theoretical analysis.

To reduce the measurement error, we take the average of elongation and restoration direction (by adding and reducing scales) results as the final results. The averaged Bragg wavelength shifts with the loaded axial force are shown in Fig. 3(b). Obviously, the Bragg wavelength shifts $\Delta \lambda_{B}$ is proportional to the applied axial force $F$. A linear fit of the data gives the slope of 0.349 , and the uncertainty is 0.012 . Then Young's modulus of the copper wire can be obtained by substituting the slope value into (4), which is $110.35 \mathrm{GPa}$. The relative error between our measured value and the nominal Young's modulus is $0.32 \%$. As a comparison, Young's modulus is measured by a CCD imaging method (ZW-YM-1, the magnification of reading microscope is 25 times, the division value is $0.05 \mathrm{~mm}$, and the line pair of CCD is 420 lines $/ \mathrm{mm}$ ) under the same conditions, and the measured Young's modulus of the copper wire is $113.68 \mathrm{GPa}$, and the relative error to nominal value is $3.34 \%$. The relative error of two methods is $3.02 \%$, which indicates that the FBG test method is feasible.

In order to evaluate the stability and repeatability of the proposed method, we perform 10 times of experimental measurements of copper wires by adding or reducing scales in the tray. The relative errors distribution is plotted in Fig. 4. The solid dots in Fig. 4 represent the average value of adding scales process and reducing scales procedure. It can be seen from Fig. 4 that the mean relative errors measured by the FBG sensor is $0.41 \%$, and the least value can reach up to $0.27 \%$. In the following experimental results, all the measured FBG reflected spectra shifts with the change of the applied axial force are corresponding to elongation direction, and the results of Bragg wavelength shifts with the loaded axial force are the average value of the two direction's results.

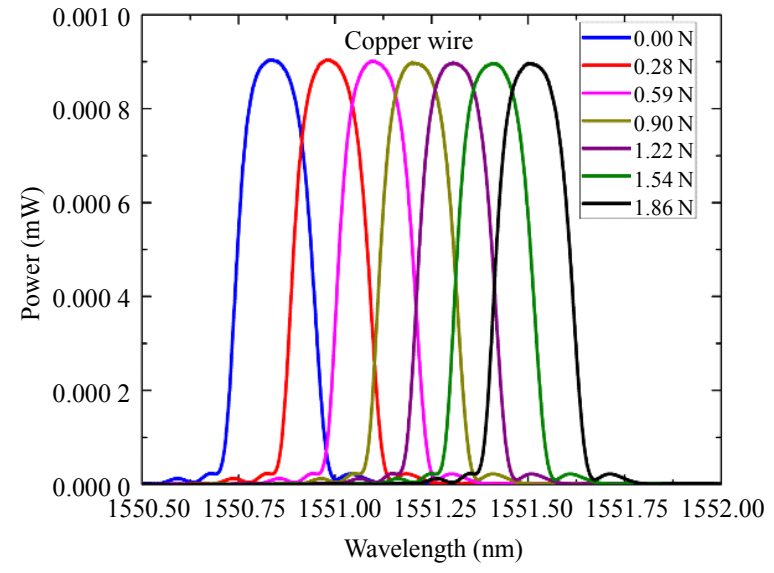

(a)

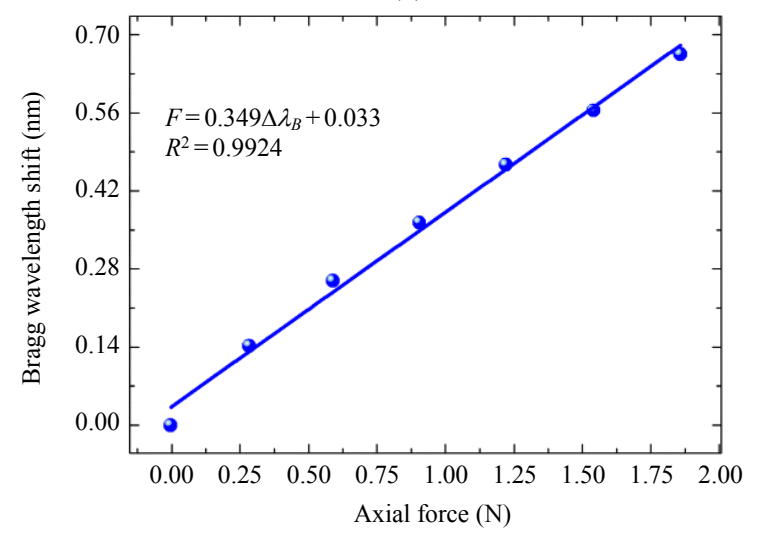

(b)

Fig. 3 Bragg wavelength of FBG changes with the loaded axial force: (a) the measured FBG reflected spectra shifts with the change of the applied axial force $F$ and (b) the Bragg wavelength of FBG responses to the variation of the loaded axial force.

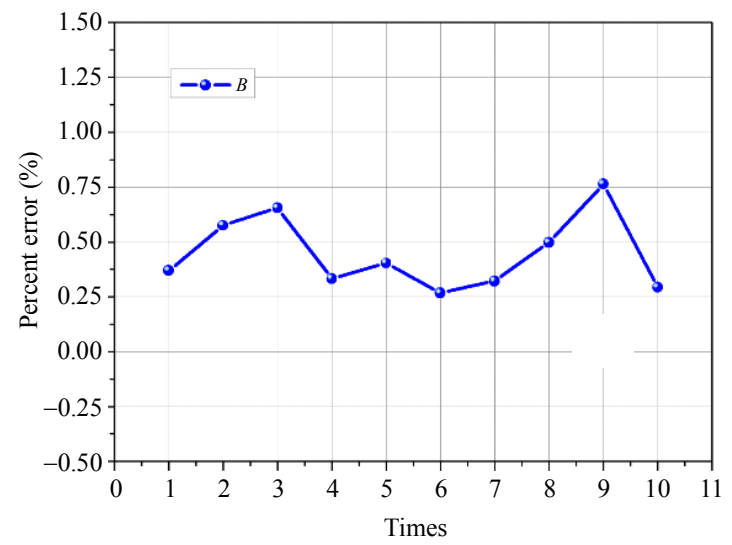

Fig. 4 Relative error distribution for adding or reducing scales process.

\subsection{Measuring Young's modulus of other metal wire}

In order to verify that the proposed scheme can be used to measure Young's modulus of various metal wires, we separately measured the Young's 
modulus of aluminum wires, nickel wires, and tungsten wires by using the proposed FBG method. In the experiments, different axial force ranges were loaded for different metal wires to ensure enough wavelength shift of FBG. The tungsten wire had least deformation property. And thus, the maximum axial force ranging from $0 \mathrm{~N}$ to $3.60 \mathrm{~N}$ was loaded on the tungsten wire in the experiment. The nominal Young's modulus of the aluminum wires, nickel wires, and tungsten wires in data sheets are $69.00 \mathrm{Gpa}, 210.00 \mathrm{Gpa}$, and $340.00 \mathrm{GPa}$, respectively.

The measured results are shown in Fig. 5. FBG reflection spectra in Figs. 5(a), 5(b), and 5(c) correspond to aluminum wires, nickel wires, and tungsten wires, respectively. Corresponding Bragg wavelengths of the FBG response to the variation of the loaded axial force are shown in Fig.5(d). The measurement of FBG reflected spectra for the three
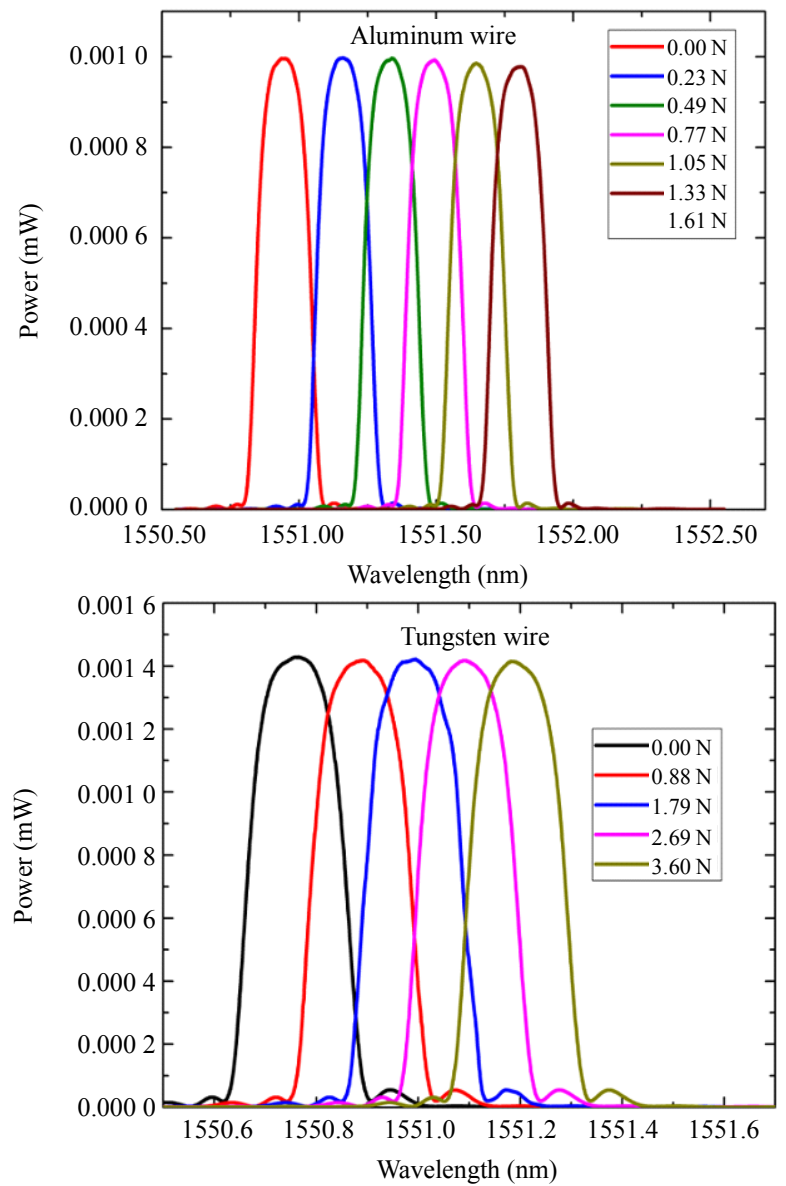

kinds of wires also show a uniform spacing change with an increase in the axial force applied, which well agrees with the theoretical analysis. The slopes of the Bragg wavelength response curves for aluminum wire, nickel wire, and tungsten wire are 0.61 [red dotted line in Fig. 5(d)], 0.31 [green dotted line in Fig. 5(d)], and 0.12 [blue dotted line in Fig. 5(d)], respectively. Then Young's modulus of three wires can be calculated as copper wire, and the values are $68.59 \mathrm{GPa}, 211.30 \mathrm{GPa}$, and $340.73 \mathrm{GPa}$, and corresponding relative errors are $0.58 \%, 0.62 \%$, and $0.21 \%$, respectively. For clear comparison, Young's moduli of the three wires are measured by the conventional CCD imaging system under the same conditions, and the measured results are listed in Tables 1 and 2. It is clear that the relative errors measured by FBG sensors are within $1.0 \%$. The relative errors between the two methods are $6.95 \%$, $3.92 \%$, and $5.36 \%$, respectively.
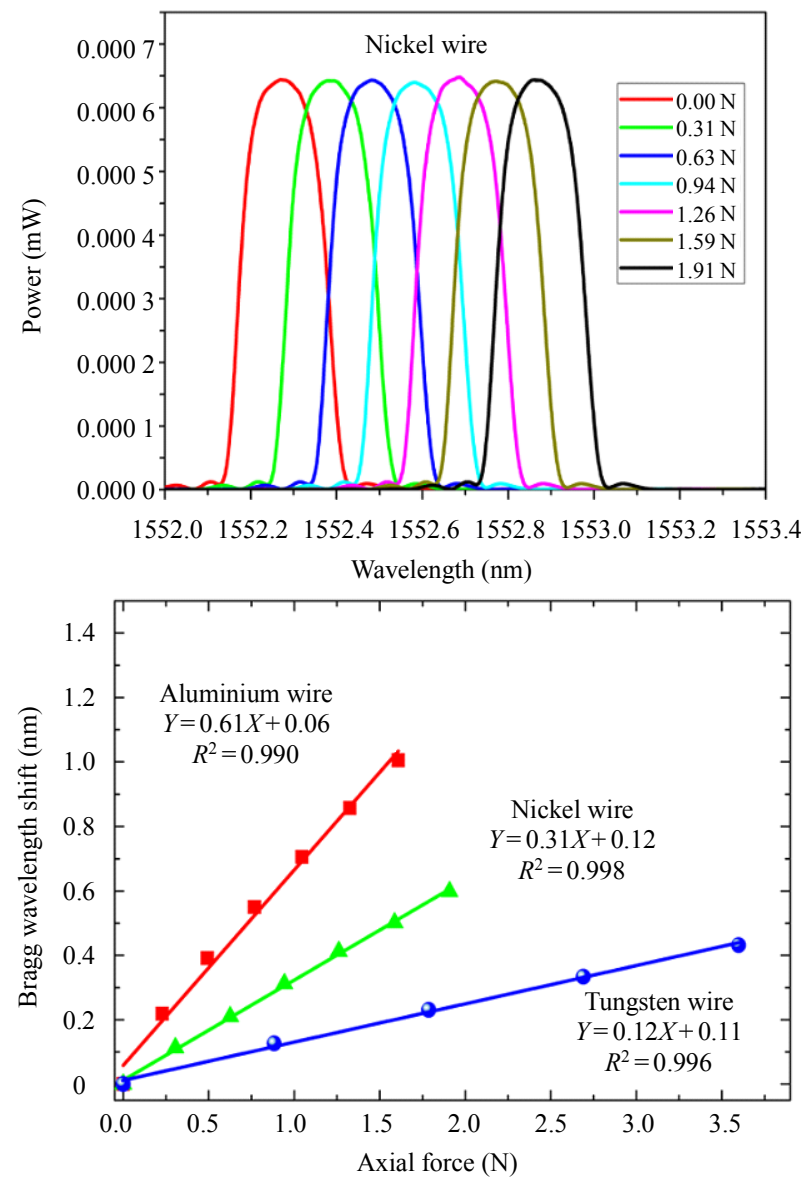

Fig. 5 FBG reflective spectra shift with the change of the axial forces: (a) aluminum wires, (b) nickel wires, (c) tungsten wires, and (d) the Bragg wavelengths of FBG response to the variation of the loaded axial force. 
Table 1 Young's modulus measured by CCD system.

\begin{tabular}{cccc}
\hline Material & Nominal value $(\mathrm{GPa})$ & $\mathrm{CCD}(\mathrm{GPa})$ & Relative error $(\%)$ \\
\hline Alum. & 69.00 & 73.36 & 7.19 \\
Nickel & 210.00 & 219.58 & 4.56 \\
Tungsten & 340.00 & 359.00 & 5.59 \\
\hline
\end{tabular}

Table 2 Young's modulus measured by FBG method.

\begin{tabular}{cccc}
\hline Material & FBG (GPa) & Relative error (\%) & $\begin{array}{c}\text { Relative error of the two } \\
\text { methods (\%) }\end{array}$ \\
\hline Alum. & 68.59 & 0.58 & 6.95 \\
Nickel & 211.30 & 0.62 & 3.92 \\
Tungsten & 340.73 & 0.21 & 5.36 \\
\hline
\end{tabular}

It must be pointed out that relatively expensive OSA is used for FBG Bragg wavelength demodulation in our experiments, but it can be replaced by other lower cost interrogation systems such as fiber Fabry-Pérot interferometers [20], wavelength division coupler [21], or unbalanced Mach-Zehnder fiber interferometer methods [12], which would make the FBG sensor more suitable for engineering application in the material selection.

\section{Conclusions}

In conclusion, a universal formula based on FBG sensor has been derived to measure Young's modulus of various metal wires. Young's modulus of copper wires, aluminum wires, nickel wires, and tungsten wires are measured by using the FBG sensor according to the formula. Compared with the CCD imaging method, the FBG method shows higher precision. All the relative errors measured by the FBG sensor to the nominal values are less than $1.0 \%$. The stability and repeatability of the proposed method have also been verified. The proposed scheme with its excellent performances may find an extensive application in the material selection with relevance in the fields of civil engineering, machine design, and development of new materials.

\section{Acknowledgment}

This work was supported by the National Natural Science Foundation of China under Grant No. 61565002, and Guangxi Province Key Research and Development Program under Grant Nos. AB17129027 and AB18221033.
Open Access This article is distributed under the terms of the Creative Commons Attribution 4.0 International License (http://creativecommons.org/licenses/by/4.0/), which permits unrestricted use, distribution, and reproduction in any medium, provided you give appropriate credit to the original author(s) and the source, provide a link to the Creative Commons license, and indicate if changes were made.

\section{References}

[1] K. O. Hill and G. Meltz, "Fiber Bragg grating technology fundamentals and overview," Journal of Lightwave Technology, 1997, 15(8): 1263-1276.

[2] M. M. Elgaud, M. S. D. Zan, A. Abushagur, and A. A. A. Bakar, "Improvement of signal to noise ratio of time domain mutliplexing fiber Bragg grating sensor network with Golay complementary codes," Optical Fiber Technology, 2017, 36: 447-453.

[3] G. Guo, D. A. Hackney, M. Pankow, and K. Peters, "Interrogation of a spectral profile division multiplexed FBG sensor network using a modified particle swarm optimization method," Measurement Science and Technology, 2017, 28: 055204-1055204-9.

[4] K. Tian, Y. L. Liu, and Q. M. Wang, "Temperature-independent fiber Bragg grating strain sensor using bimetal cantilever," Optical Fiber Technology, 2005, 11(4): 370-377.

[5] L. Jin, W. G. Zhang, H. Zhang, B. Liu, C. J. Zhao, Q. C. Tu, et al., "An embedded FBG sensor for simultaneous measurement of stress and temperature," IEEE Photonics Technology Letter, 2006, 18(1): 54-156.

[6] Y. K. Chamorovskiy, O. V. Butov, A. O. Kolosovskiy, S. M. Popov, V. V. Voloshin, I. L. Vorob'ev, et al., "Metal-coated Bragg grating reflecting fiber," Optical Fiber Technology, 2017, 34: 30-35.

[7] G. Allwood, G. Wild, A. Lubansky, and S. Hinckley, "A highly sensitive fiber Bragg grating diaphragm pressure transduce," Optical Fiber Technology, 2015, 25: 25-32.

[8] W. Liang, Y. Y. Huang, Y. Xu, R. Lee, and A. Yariv, "Highly sensitive fiber Bragg grating refractive index sensors," Applied Physics Letters, 2005, 86(15): 151122-1-151122-3.

[9] J. H. R. Oliveira, S. Aristilde, G. Chesini, M. A. R. Franco, R. N. Nogueira, and C. M. B. Cordeiro, "Bragg gratings in surface-core fibers: refractive index and directional curvature sensing," Optical Fiber Technology, 2017, 34: 86-90.

[10] Y. S. Zhang, W. G. Zhang, Y. X. Zhang, S. Wang, L. Yu, and T. Y. Yan, "Simultaneous measurement of curvature and temperature based on LP 11 mode Bragg grating in seven-core fiber," Measurement Science and Technology, 2017, 28(5): 
055101-1-055101-6.

[11] G. Allwood, G. Wild, A. Lubansky, and S. Hinckley, "A highly sensitive fiber Bragg grating diaphragm pressure transducer," Optical Fiber Technology, 2015, 25: 25-32.

[12] X. Y. Deng, G. H. Chen, Q. X. Peng, Z. R. Li, J. H. Meng, and J. Liu, "Research on the fiber Bragg grating sensor for the shock stress measurement," Review of Scientific Instrument, 2011, 82(10): 103109-1-103109-4.

[13] M. M. J Treacy, T. M. J. Ebbesen, and J. M. Gibson, "Exceptionally high Young's modulus observed for individual carbon nanotubes," Nature, 1996, 381: 678-681.

[14] A. Krishnan, E. Dujardin, T. W. Ebbesen, P. N. Yianilos, and M. M. J. Treacy, "Young's modulus of single-walled nanotubes," Physical Review B, 1998, 58(20): 14013-14019.

[15] I. Maruyama, H. Sasano, Y. Nishioka, and G. Igarashi, "Strength and Young's modulus change in concrete due to long-term drying and heating up to $90{ }^{\circ} \mathrm{C}, "$ Cement and Concrete Research, 2014, 66: 48-63.

[16] W. W. Quan, J. Kang, L. Yang, and J. H. Hu,
"Young's modulus measurement of metal beams based on fiber Bragg grating," Laser \& Optoelectronics Progress, 2016, 53: 040604-1-040604-6.

[17] Y. Zou, X. P. Dong, G. B. Lin, and R. Adhami, "Wide range FBG displacement sensor based on twin-core fiber filter," Journal of Lightwave Technology, 2012, 30(3): 337-343.

[18] D. J. Hill and G. A. Cranch, "Gain in hydrostatic pressure sensitivity of coated fiber grating," Electronics Letters, 1999, 35(15): 1268-1269.

[19] A. Kersey, M. A. Davis, H. J. Patrick, M. Leblanc, K. Koo, C. G. Askins, et al., "Fiber grating sensors," Journal of Lightwave Technology, 1997, 15(8): 1442-1463.

[20] W. Z. Huang, W. T. Zhang, and F. Li, "Swept optical SSB-SC modulation technique for high-resolution large-dynamic-range static strain measurement using FBG-FP sensors," Optics Letters, 2015, 40(7): 1406-1409.

[21] M. A. Davis and A. Kersey, "All-fiber Bragg grating strain-sensor demodulation technique using a wavelength division coupler," Electronics Letters, 1994, 30(1): 75-77. 